welche das integrativ konzipierte Betriebsrätesystem außer Kraft setzen und dafür die lösende Aussperrung funktionalisieren will, repräsentiert die BDA eine Kapitalgruppe, die dieses System anerkennt, die suspendierende Aussperrung aber für die Veränderung der Form des Tarifverhandlungssystems (Zentralisierung) instrumentalisiert."s

Indem das BVerfG die Verfassungsbeschwerde verworfen hat unter Ausklammerung sämtlicher in der Aussperrungsdiskussion strittiger Probleme, hat es der politischen Diskussion über Zulässigkeit und Bedeutung der (suspendierenden) Aussperrung keine Schranken gesetzt und zugleich den Unternehmern eine Absage erteilt, die sich lästiger Betriebsräte unter Umgehung des BerrVG entledigen wollen. ${ }^{6}$

Rainer Erd

\title{
Zur Diskriminierung alleinstehender Mütter und Väter im Steuerrecht
}

Vorbemerkung

Dieser Beitrag thematisiert das Problem der Diskriminierung alleinstehender Mütter und Väter in der BRD exemplarisch für die Frage des sog. , Ehegatten $ヶ$ Splitting im Steuerrecht. Das Splitting-Verfahren ( $\$ 32 a$ Einkommensteuergesetz) begünstigt in der herkömmlichen Auslegung die ,Hausfrauen-Eher und ordnet sich so in das gesetzgeberische Leitbild der nichterwerbstätigen Ehefrau ein. Die Begünstigung besteht darin, das Einkommen des allein Erwerbstätigen rechnerisch auf beide Ehegatten aufzuteilen und mit einem geringeren Steuersatz zu belegen (Progressionsminderung).

" Das Gutachten von Zöllner für die BDA bestätıgt die These. Historısch neu für die konservatıve urıstısche Aussperrungsdiskussıon ist, daß Zöllner sich ausschließlich mit den von linken Arbeitsrechtlem (hauptsächlich mit Michael Kittner, dem Justutiar der IG Metall, vgl. GewMH 1973, 9I) vorgetragenen Argumenten zur verfassungsrechtlichen Unzulässıgkeit der Aussperrung insgesamt auseınandersetzt, damı über wette Strecken den Boden dogmatıscher Diskussıon verlassen muß und beı der Begründung der Zulässıgkeıt der Aussperrung nur noch mühsam seıne politischen Intentıonen, das Streikrecht zum weitgehend untauglichen Mittel gewerkschattlicher Politik zu degradieren, hinter normatrven Figuren verbergen kann. ». . e ene Verfassungsgarantie der Aussperrung unter Parıtärsgesich sspunkten (könnte) entfallen. ., wenn der Gesetzgeber durch entsprechende Regelungen des Streikrechts sıchersrellt, daß die Bestreikung einzelner Unternehmen, und die Durchführung von Schwerpunktstreiks oder Streiks mut relatw gerınger Fläche unterbleibt. Mit der Beschränkung der Gewerkschaftsseite auf großflächige Angriffsstreiks würden unter Paritätsgestchtspunkten auch im Hinblick auf die inhaltliche Richtıgkett des ausgehandelten Vertrages wohl keıne gewıchtıgen Bedenken bestehen.* (Zöllner, a. a. O., S. 58; Hervorhebungen von mur, R. E.).

Daß es den Großunternehmen um eine bestimmte Form des Tarifverhandlungssystems geht, wird auch daraus deutlich, daß Gesamtmetall gegen die "Neutralitätsanordnung a der Bundesanstalt für Arbett vom 22. 3. 1974, wonach auch solche Arbeitnehmer Arbeitslosengeld erhalten, die wegen enes Streiks in einem benachbarten Tarifbezırk der gleichen Branche zeırwesse arbertslos werden, Verfassungsbeschwerde erhoben hat. Gesamtmetall will mit der Verfassungsbeschwerde erreichen, daß die IG Merall im Streikfall an die betreffenden Lohnabhängıgen Streikgelder zahien muß und damıt in ihrer Streikfähıgkeıt engeschränkt wrrd; vgl. FR v. 23. 7. 1974 .

16 Diese grundsätzliche Bedeutung der Entscheıdung des BVerfG wird weder in der Besprechung von Wolfgang Schneider/Michael Schoden, Ein interessantes Ureit zur Aussperrung, Die Quelle, Nr. 5/1975, S. 217 f. noch in der Anmerkung von e. e., Bundesverfassungsgencht: Aussperrung von Betrebsratsmitgliedern grundsätzlich nur suspendierend, Rote Robe, $\mathrm{Nr} .4 / 1975$, S. $164 \mathrm{f}$. gesehen. Die beiden Stellungnahmen gehen nur summarısch auf die Relevanz der Entscheidung für Betnebsräte ein, der politısche Stellenwert für die gewerkschaftliche Aussperrungsdiskussıon hıngegen wird nicht untersucht. 
Gegen die beschriebene Diskriminierung beginnen sich alleinstehende Mütter und Väter zu wehren. Ihr Ziel ist die Beseitigung des Ehegatten-Splitting, zumindestens aber die steuerrechtliche Gleichbehandlung alleinstehender Elternteile gegenüber verheirateten. Der Verband alleinstehender Mütter e. V. führt eine Kampagne für diese Gleichbehandlung durch: möglichst viele alleinstehende Elternteile sollen auf einer Einkommensbesteuerung nach dem Splitting-Tarif bestehen; in Musterprozessen soll dieses Ziel verfolgt werden.

I.

Das Familienrecht ist in Bewegung. Diese Diagnose gilt nicht nur für den engeren Bereich des $B G B, 4$. Buch, sondern für die zunehmend wichtigeren Materien des familienbezogenen öffentlichen Rechts: Für Sozialversicherungsrecht ebenso wie für Steuerrecht, für Ausbildungsrecht ebenso wie für Jugendhilferecht. Die Kraft, aus der die Bewegung ihren Arem schöpft, auch gegen anhaltende Widerstände anzurennen, haben wir an anderer Stelle zu benennen versucht'. Dort ist dargestellt, daß nicht beliebige Reformen auf beliebige Wünsche beliebiger Gruppen anstehen, sondern daß gesellschaftliche Norwendigkeiten zu verhandeln sind, deren Vernachlässigung zu möglicherweise lange nicht wieder behebbarer Gefährdung der Reproduktion der Gesellschaft auf dem erreichten Produktivitätsniveau führen könnte. Die politische Führung des Landes - und da sind alle parlamentarisch vertretenen Parteien einbegriffen - geht mit dieser Bedrohung außerordentlich leichtfertig um, wenn sie etwa die Erstellung eines Jugendhilfegesetzes aus konjunkturellen Erwägungen zurückstellt - krasses Beispiel einer gleichzeitig auf Bedarfsbefriedigung und Konjunkturbeeinflussung zielenden Ausgabenpolitik des Staates², bei dem kurzsichtig der aktuellen ökonomischen Situation der Vorrang eingeräumt wird unter Aufgabe der wesentlichen Staatsfunktionen, das bürgerliche Allgemeininteresse durchzusetzen, d. h. zentral, langfristig zu planen.

Nicht nur am - vorläufigen - Schicksal der Reform des Jugendhilferechts wird deutlich, daß die Erkenntnis in die Notwendigkeit bestimmter gesellschaftlicher, d. h. staatlicher Aufgabenerfüllung keineswegs ihre Durchführung garantiert', daß das Notwendige nicht auch das Selbstverständliche ist, sondern noch erstritten werden muß, durchgesetzt gegen chronische und strukturbedingte Finanzkrisen des Staates. So erklärt sich die Ungereimtheit, daß die große Novelle des Einkommensteuergesetzes von $1974^{4}$ unverändert das progressions- und steuerlastmindernde Ehegatten-Splitting fortschleppt (\$32a Abs. s EStG) - Ungereimtheit deshalb, weil sich in der Familienrechts-Reform-Diskussion, die den Zusammenhang von rechtlicher und gesellschaftlicher Entwicklung ernst nimmt und nicht nur die Weisheiten der Vergangenheit unbeeindruckt von dem Problem ihrer fortdauernden Adäquanz wiederholt, umfassend und eindeutig herausgeschält har, daß das öffencliche (Förderungs-)Interesse nicht auf den Tatbestand,Ehe<, sondern auf Fortpflanzung und Kindererziehung konzentriert ist. Das gilt für die Novellierung der privaten Unterhaltspflichten nach Auflösung der Ehe' ebenso wie für die Reform des familienrele-

- Gunnar Heinsohn/Rolf Kneper, Theorie des Familienrechts: Geschlechtsrollenaufhebung, Kindesvernachlässıgung, Geburtenrückgang, 1974 .

- Vgl. ausführlicher Piduch, Bundeshaushaitsrechr, Bd. I, Stand Mai 1974, Einführung 9.

- Vgl. etwa Offe, Strukturprobleme des kapitalistuschen Staates, 1972, bes. S. 33 ff.

+ BGBI I, S. 2165.

- Erstes Gesetz zur Reform des Ehe- und Familienrechts BR/DS 260/73. 
vanten Sozialversicherungsrechts: Zentraler Unterhaltsgrund soll die Erziehung von Kindern sein (vgl. $\$ 157$ I BGB-E), und die pluralistisch zusammengesetzte Eherechtskommission beim Bundesministerium für Justiz entschied, nur der Kinder aufziehenden (Ehe-)Frau sei der Zugang zu Systemen sozialer Sicherung öffentlich zu finanzieren; für die kinderlose Nur-Hausfrau ${ }^{6}$ soll es bei freiwilliger und ehegatten-finanzierter Sozialversicherung bleiben', wie sie inzwischen mir dem Rentenreformgesetz von $1972^{8}$ eingeführt worden ist. Das >Bekenntnis«, „daß der Rückzug der Ehefrau auf die Führung des ehelichen Haushalts legitım, der Rückzug der Mutrer auf die Erziehung der Kinder und die Führung des Familienhaushalts darüber binaus $z u$ fördern «" sei, entsteht aus dieser Differenzierung: daß die Entscheidung, einen kinderlosen Ehepartner als lediglich haushaltsführenden zu shalten`, privat sei und auch privat zu finanzieren sei, daß aber die nicht erwerbstätige Mutter eine gesellschaftliche Aufgabe erfülle, die gesellschaftlich zu honorieren sei. Die dem individuellen Reproduktions-Bedürfnis von Privateigentümern entsprechende Ehedefinition noch des preußischen ALR, nach der "Hauptzweck ... der Ehe die Erzeugung und Erziehung von Kindern « (II I $\$ I ALR) ist, hat die Verallgemeinerung der Lohnarbeit inzwischen weitgehend überholt, ohne daß Gebären und Aufzucht von Kindern als Notwendigkeit entfallen wären: über Privatheit sich herstellende Gesellschaftlichkeit ist im Kern unmittelbarer Gesellschaftlichkeit gewichen.

Politik und Gesetzgebung nehmen nun nicht in rational planendem Zugriff solche gesellschaftstheoretische Erkenntnis auf, sondern reagieren in der Regel - wenn überhaupt - auf Erscheinungen, die aus der Entwicklung selbst resultieren müssen. Die Klarheit, mit der etwa die Motive zum Bürgerlichen Gesetzbuch den Zusammenhang von ökonomischem Interesse und Zuwendung für Kinder festhielten, als es um die Ausgestaltung der Position der Nichtehelichen-Mutter ging ${ }^{10}$, gehört zu den Ausnahmen rechtspolitischer Argumentation. Die Erscheinungen aber stellen sich unerbittlich ein und werden als Bedrohung wahrgenommen: als zunehmende Kindesvernachlässigung, als Leistungsabfall, als Verwahrlosung, als Geburtenrückgang, aber auch in Forderungen von Jugendlichen an den Staat: nach Ausbildungsreform, nach öffentlicher Finanzierung der Ausbildung, nach Jugendzentren.

Indem Familienrechtsreform die Erscheinungen aufnimmt, verarbeiter und so der Faktizität der gesellschaftlichen Bewegung folgt, indem sie zunächst zwischen Ehefrau und Mutter differenziert, beginnt sie gleichzeitig die Entwicklung zu bewältigen, welche die Substanz der patriarchalischen Kleinfamilie mit ihrer strengen Geschlechtsrollen-Trennung ausgehöht hat, eine Substanz, die durch Propaganda nur eine Weile ersetzt werden kann". Die Aufhebung der Geschlechtsrollen

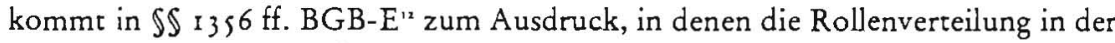
Ehe nicht mehr geschlechtsspezifisch festgelegt ist, in der nur noch von privat sich einigenden Ehegatten, nicht aber mehr von ,Mann und ,Frau die Rede ist.

\footnotetext{
- Dieser Begriff wırd hier ohne pejoratıve Absıchı benutzt, da der von Pross eingeführte alternatıve Begriff der Familien-Frau für's Steuerrecht nıcht paßt, das beı der Ehegattenprogressıon nıcht an die Exıstenz von Kindern anknüpft. Vgl. Pross, Die Wirklichke1t der Hausfrau, 1974, S. 64 ff.

, Eherechtskommission beim Bundesministerium der Justız, Vorschläge, 7. Teil, 1972.

s BGBI I S. 1969 .

- Zacher, Empfiehlt es sich, die gesetzlichen Vorschriften über die sozıale Sicherung der nıchtberufstätıgen Frau während und nach der Ehe, insbes. Im Fall der Scheidung, zu ändern? Sitzungsberichte zum 47. Deutschen Juristentag, 1968, S. O 12 (Sperrung vom Verfasser); vgl. etwa auch Ruland, Die Stellung der Frau in der Sozıalversicherung, FamRZ I975, 144.

10 Mugdan, Die gesamten Matenalien zum Bürgerlichen Gesetzouch für das Deutsche Reich, IV Band, Familienrecht, 1899 , S. $453 \mathrm{ff}$.

"Vgl. ausführlich G. Hetnsohn/R. Kneper a. a. O.

"Siehe FN s.
} 
Die analytische Trennung von legalisierter Geschlechtsgemeinschaft , Ehe und Zeugung/Gebären/Aufzucht von Kindern hat ihre unabänderliche Grundlage in der Geschichte der bürgerlichen Gesellschaft selbst. Sie wird in den durchgeführten und anstehenden Gesetzgebungsplänen, wenn auch mit Zögern und Brüchen, aufgenommen: Die kinderlose Ehe verliert vollends den Charakter des ,Offentlichen stellt sich als die rein private Angelegenheit der Partner dar, welche sie nach dem lange erfolglosen Petitum liberaler und sozialistischer Wissenschaftler immer schon sein sollte; entsprechend gilt die Versorgung der kinderlosen Ehepartner als Privatsache, deren Finanzierung ihrer Abrede überlassen ist. Die Reproduktion der Bevölkerung dagegen ist grundsätzlich als öffentliche Aufgabe anerkannt, was sich nicht nur in der Zunahme gesellschaftlicher Erziehung dokumentiert, sondern gerade auch in der prinzipiellen Bereitschaft, die individuelle Bezugsperson, d. h. die Mutter oder den Vater, durch Erziehungsberatung und finanzielle Zuwendung zu unterstützen. Pläne zur Ausdehnung des Mutterschutzes, zur Einführung eines Erziehungs- und Mutrergeldes verweisen darauf ${ }^{\prime \prime}$. Die in diesem Zusammenhang häufig beteuerte Bevorzugung individueller vor gesellschaftlicher Erziehung gerade kleiner Kinder lebt von der Hoffnung auf die Sozialisationspotenz der Familie, die gesellschaftlichen Einrichtungen nicht zugetraut wird ${ }^{14}$. Während diese Hoffnung Gebär- und Aufzuchtsförderungsprogramme noch weitgehend an die Familie zu binden versucht, und während auch noch Kinder zum größten Teil in Familien geboren und aufgezogen werden, weisen doch schon Murterschurz- und Erziehungsgeldgesetzgebung über die Familie hinaus, indem an das Kind angeknüpft wird unabhängig davon, ob es in >vollständigen oder sunvollständigen Familien aufwächst. Die Schutzformel des Art. 6 Abs. IV GG konkretisiert sich, wobei gleichzeitig die geschlechtsspezifische Formulierung überholt wird: der alleinstehende Vater wird in die ,Fürsorgec - Verpflichtung einbezogen.

II.

Die Reform des Einkommensteuerrechts von 1974 vernachlässigt diese für andere Gesetzesvorhaben breit diskutierten Notwendigkeiten in aller Eindringlichkeit, indem sie im Ehegatren-Splitring fortgesetzr die Ehe öffentlich subventioniert, ohne die Förderung an das Vorhandensein von Kindern zu binden und indem sie - nach der Wort-Interpretation des $₫ 32 a$ Abs. V EStG - alleinstehende Bezugspersonen, Elternteile, von der Begünstigung ausnimmt. Die für Ehe-, Kindschafts- und Sozialversicherungsrecht fruchtbar gemachte theoretische Einsicht verkehrt sich in ihr praktisches Gegenteil: die sPrivat-Angelegenheit< Ehe kommt in den Genuß öffentlicher, von der Allgemeinheit finanzierter Subvention, welche der öffentlichen Angelegenheir Kindererziehung in der unvollständigen Familie verwehrt bleibr.

Die Entwicklung der Ehegattenbesteuerung belegt, daß sie sich - aus konjunkturellen, weltanschaulichen und bevölkerungspolitischen Gründen - gegen den Prozeß der Lohnarbeit von (Ehe-)Frauen stemmt, ohne ihn freilich aufhalten zu können, und »die Ehefrau ins Haus zurückzuführen «'s versucht: Die NSDAP-Regierung führt erstmals 1936 die obligatorische Zusammenveranlagung von Ehegatten ein,

1) Vgl. zuletzt: Berıcht über die Lage der Familie in der Bundesrepublik - Zweiter Familienberıchr - BT/DS $7 / 3502$.

14 Zu einer Analyse der strukturellen Schwierıkeiten vergesellschafteter Kleinkundererziehung vgl. neuerdings Gunnar Hetnsohn/Barbara M. C. Kneper, Theorıe des Kindergartens und der Spielpädagogik, 1975 .

" Nachwerse be1 G. Heinsohn/R. Knueper a. a. O., S. 106 f./122; BVerfGE 6, SS (1957). 
nach der die Einkommen Erwerbstätiger addiert werden mit der Konsequenz erhöhter Progression. Nachdem die darin liegende Diskriminierung fakrisch der erwerbstätigen Ehefrau 1941 aufgehoben wird, um den von Männern kriegsbedingt 'gesäuberten^ Arbeitsmarkt zu füllen, führt eine CDU-Regierung sie I95I wieder ein ${ }^{16}$ Auf das Urteil des Bundesverfassungsgerichts vom 17. 1. 1957, das auf Verfassungswidrigkeit der Diskriminierung erkennt ${ }^{16}$, folgt die Novellierung zum Ehegatten-Splitting. Sie vermeidet die offene Diskriminierung der erwerbstätigen Ehefrau gegenüber dem Ledigen, erhält sie aber gegenüber der Nur-Hausfrau, deren Nichterwerbstätigkeit im Millionärsgattinnen-Effekt ${ }^{17}$ progressionsmindernd honoriert wird. Diese Regelung ist beinahe wortidentisch durch die Steuer-Reform geschleppt worden.

Mag nun noch mit einer Reihe von gegenüber der ökonomischen Struktur kontingenten Gründen erklärbar sein, daß auf Eheschließung hin erzogen wird, daß Ledige bestraft und dem Leitbild `Hausfrauen-Eher Folgende belohnt werden, um Geschlechtsgemeinschaften auf $D$ auer zu stellen, zu legalisieren, auf deren vielfältige Funktionen zu hoffen, und mag die partiell zähe Verteidigung der Ehe unabhängig von Kindern durch Frauen wie Männer auf subjektive, psychische Dispositionen rechnen können, welche die objektive Struktur der Gesellschaft (noch) überdau$e^{-18}{ }^{18}$, so ist doch die Grenze des Verständnisses überschritten, wenn Verfassungsnormen sich mit gesellschaftlicher Struktur verbünden: Die Anerkennung von Kindergebären und -aufzucht als öffentliche und öffentlich zu fördernde Aufgabe hat zwar ihre Kraft aus der wachsenden Einsicht in gesellschaftliche Notwendigkeiten gezogen, ohne sich intensiv auf Verfassungsnormen zu stützen. Das heißt aber keineswegs, daß nicht das Grundgesetz verletzt wäre, wenn alleinstehende Mütter/ Väter gegenüber Ehepartnem, die auch Mütter und Väter sein mögen, zu rückgesetzt werden. Nicht nur sichert Art. 6 Abs. IV GG Müttern (über die geschlechtsspezifische Formulierung ist die Entwicklung teilweise, d. h. abgesehen von biologischen Besonderheiten, hinweggegangen) die besondere Fürsorgepflicht der Gesellschaft zu, nicht nur billigt Art. 6 Abs. V GG die materielle Gleichheit der nicht-ehelichen Kinder, die sich in wichtigen Teilen allein über Unterstützung von Elternteilen erreichen läßt, sondern auch der allgemeine Gleichheitssatz (Art. 3 GG) verbietet jede Diskriminierung. Die Folgerung nämlich, daß ,Ungleiches ungleich، behandelt werden könne, ist unter keinen Umständen in der Weise zu konkretisieren, daß in Ungleichheit resultierende faktische Sonderbelastungen zusätzlich noch als Grundlage für gesetzliche Sonderbelastung herangezogen wird. Wenn - was unbestritten ist - Verfassungsrecht die Diskriminierung Lediger wie alleinstehender Elternteile verbietet, wenn weiterhin die gesellschaftspolitische Einsicht fordert, Eltern(-teile) eher 'gleicher $z u$ behandeln als Kinderlose, dann kann die Wortinterpretation eines Reform-Gesetzes keinen Bestand haben, soweit sie verfassungsrechtliche und gesellschaftspolitische Maßstäbe verletzt.

Eine Wortinterpretation des $\ 32 \mathrm{E}$ EStG müßte zu dem Ergebnis führen, die Vorteile des Ehegatten-Splittings auf den Tatbestand der Ehe zu beschränken, d. h. sie nicht von der Existenz von Kindern, aber auch nicht von einer Situation abhängig zu machen, in der alleinstehende Mütter/Väter neben der Haushaltsführung und Erziehung erwerbstätig sind, in der also im Ergebnis die nach dem Leitbild 'Hausfrauen-Eher normalerweise getrennten Rollen in einer Person konzentriert sind. Mit dieser Rollen-Vielfalt erfüllen sich in einer Person die Voraussetzungen,

is BVerfGE 6, 55/79 (1957).

"Vgl. insgesamt Mennel, Teilgutachten Steuerrecht in: Löwısch/Gitter/Mennel, Welche rechtlichen Maßnahmen sınd vordrınglich, um die tatsächliche Gleıchstellung der Frau mit den Männern im Arbeirsleben zu gewährletsten? Gutachten D zum so. Deutschen Juristentag 1974

is G. Heinsohn/R. Knieper a. a. O., S. I 66 ff. 
die heute als Begründung für die steuerliche Bevorzugung der Nur-Hausfrau gelten, nachdem die schon von der NSDAP verfolgte Frau-in-die-Küche-Politik، gegen das Grundgesetz verstößr: Mir der Gleichberechtigungsgesetzgebung von I957 wurde zum ersten Mal qua Legal-Definition anerkannt, daß die Haushaltsführung als Arbeit, als der Erwerbstätigkeit im Prinzip gleichwertige Unterhaltsleistung zu charakterisieren sei. $\$ I 360 Satz 2 BGB normiert, daß die Führung des Haushalts Arbeit sei, mit der die Unterhaltsverpflichtung der Frau in der Regel abgegolten werde. Inzwischen besteht weitgehender Konsens über diese Einschätzung der Haushaltstätigkeit, der nun nicht nur etwa die Reform des Sozialversicherungsrechts bestimmt, sondern gerade auch das Ehegatten-Splitting auf eine korrekte Verfassungsgrundlage gestellt hat. Völlig unbestritten und durch empirische Forschung bestätigt ${ }^{\circ}$ ist auch, daß die Kombination von Haushaltsführung, Kinderaufzuchr und Erwerbstätigkeit in einer Kombination von Belastungen resultiert. Während nun in der >Hausfrauen-Ehe die im Splitting akzeptierte Möglichkeit besteht, die Lasten der Haushalts- und Erwerbstätigkeit zu trennen, sind alleinstehende Mütter/Väter in der Regel darauf verwiesen, Haushalts- und Erwerbstätigkeit sowie Kindererziehung allein durchzuführen. Die Belastung verringert sich auf diese Weise nicht. Eine solche Person ist Bezugsperson, Erzieher und Erwerbstätiger, verdient also zu versteuerndes Einkommen und leistet zusätzlich zwar nicht entlohnte, aber für Ehegatten zu Steuerersparnis einsetzbare Arbeit. Damit erfüllt diese Person substantiell eben die Voraussetzungen, welche allein zur Begründung des Ehegatten-Splitting taugt: sie ist ,Ehegatten<.

Daraus folgt, daß der Gesetzgeber zwar frei ist, den Gesamtkomplex der Ehegattenbesteuerung nach politischen Erwägungen zu gestalten, die ideologiebefrachtet auch hinter gesellschaftliche Entwicklungen und Notwendigkeiten zurückbleiben können, daß also gesetzgeberische Handlungsfreiheit die Alternative der Katastrophe einschließt, daß aber die Handlungsmöglichkeit eingeschränkt ist, wo die Reaktion auf gesellschaftliche Entwicklung durch Verfassungsnormen in Bahnen gelenkt ist. Während es also wohl allein in der Macht des Gesetzgebers steht, das gesamte System des Ehegatten-Splitting auf dem Hintergrund der Vorwürfe von Überholtheit, Ideologiebefrachtung und Irrationalität neu zu ordnen, muß das Verfassungsgebot unmittelbar in die aktuelle Auslegungspraxis eingehen. Verfassungskonform aber wäre nur eine Interpretation, welche unter den Begriff, Ehegatten< im Sinne des $\$$ 32a Abs. V EStG jedenfalls auch die alleinstehenden Mütter/Väter subsumierte, welche die als normal unterstellte Rollentrennung in ihrer Person aufheben und vereinen. Die nach dem Wortlaut vorausgeserzte Geschlechtsunterschiedenheit hindert eine solche Interpretation nicht, da das Fortschleppen geschlechtsspezifischer Anknüpfung weder dem Stand des Grundgesetzes noch dem moderner Gesetzgebungsvorhaben entspricht; die ebenfalls eindeutig geforderte Personenmehrheit aber verschlägt ebenfalls nicht, da zum einen der Zwang zu verfassungskonformer Auslegung sich auch gegen eindeutigen Wortlaut durchsetzt, und da zum anderen die gesetzestechnische, aus älteren Bestimmungen ohne weiteres übernommene Formulierung gegenüber eindeutigen gesellschaftswissenschaftlichen Einsichten und politischen Absichten zurückweicht. In den Aussagen von Mehrfachbelastung und Rollenvielfalt ist darüber hinaus ein Angebor gemacht, den Plural in ,Ehegatten، durch die >Pluralisierung، des Individuums aufzunehmen.

Entstehende Steuereinnahmen-Verluste müßte der Gesetzgeber auszugleichen suchen, wobei sich zunächst anböte, die Gewährung der Splitting-Möglichkeit an das Vorhandensein von Kindern zu binden.

Rolf Knieper

19 Vgl. etwa Pross, Benchr über eıne Untersuchung zur Situation erwerbstätıger Frauen un der Bundesrepublik (erste vorläufige Auswertung 1972), erstellt um Auftrag der Zeıtschrift >Brıgıtre. 